\title{
Pretreatment platelet/lymphocyte ratio (PLR) is a significant predictive marker for EGFR targeted therapy in patients with non-small-cell lung cancer
}

\section{Kejun Liu}

Dongguan People's Hospital

\section{Weiwei Zhang}

the fifth people`s hospital of chengdu

Qinquan Tan

Dongguan People's Hospital

\section{Guanming Jiang}

Dongguan People's Hospital

Jun Jia ( $\nabla$ jiwen6465@126.com )

Dongguan People`s Hospital https://orcid.org/0000-0003-1721-2723

\section{Research article}

Keywords: Non-small-cell lung cancer (NSCLC); Epidermal growth factor receptor (EGFR); Targeted therapy; Platelet/lymphocyte ratio (PLR).

Posted Date: August 14th, 2019

DOl: https://doi.org/10.21203/rs.2.12821/v1

License: (c) (i) This work is licensed under a Creative Commons Attribution 4.0 International License. Read Full License 


\section{Abstract}

Non-small-cell lung cancer (NSCLC) with epidermal growth factor receptor (EGFR) gene mutation is benefited from targeted therapy. There is no other superior predictive factor for targeted therapy except EGFR in the clinical. In this study, we analyzed the effect of pretreatment platelet/lymphocyte ratio (PLR) in NSCLC patients. In the present study, a total of 96 patients with EGFR mutations were included in this study. All patients received EGFR targeted therapy, until disease progression, unacceptable toxicity or other factors. The following evaluation was conducted about 3 days before initial treatment: detailed clinical history, physical examination, radiographic results, pathological diagnosis and laboratory parameters including complete blood cell counts and albumin levels. We found that pretreatment PLR is significantly associated to the PFS of NSCLC patients with EGFR targeted therapy. Patients in the PLR $\geq 190$ group had shorter PFS than those in the PLR $<190$ group $(P=0.009)$. Furthermore, the 1 -year PFS rate in the $P L R \geq 190$ group were inferior to the low value group $(P=0.016)$. Multivariate analysis confirmed the role of PLR on predicting the efficacy of targeted therapy for advanced NSCLC. In addition, we found that PLR was also an predictive biomarker for grade 3/4 adverse events of diarrhea. In conclusion, high pretreatment PLR was an independent indicator for predicting a poor PFS for NSCLC patients receiving EGFR-TKIs treatment. Further studies are needed to identify the impact of PLR on results of EGFR-TKIs treatment.

\section{Background}

Lung cancer, the most frequent cancer diagnosed each year, is commonly classified into small-cell lung cancer (SCLC) and non-small-cell lung cancer (NSCLC) [1,2]. Approximately $80 \%$ of lung cancer was diagnosed as NSCLC and $40-50 \%$ cases harboring epidermal growth factor receptor (EGFR) mutations in Asian patients [3-5]. NSCLC was closely associated with inflammation and chronic infection [6,7]. The tumor microenvironment of lung cancer is composed of tumor cells, inflammatory cells, fibroblasts, among others. It is postulated that tumor progression may be promoted by a variety of inflammatory factors, which may eventually affect the chemotherapeutic efficacy of cancer [8].

For patients treated with EGFR tyrosine kinase inhibitors (EGFR-TKIs), it is still unknown whether inflammatory factors would effect the antitumor efficacy of targeted drugs. Till date, EGFR mutation status is still the only effective predictor for advanced NSCLC patients with EGFR targeted therapy. Hence, to find superior predictive biomarkers of systemic inflammatory response for patients receiving targeted therapy is very meaningful for oncologist.

Over the last decade, hematological inflammatory response markers such as platelet/lymphocyte ratio (PLR) and C-reactive protein/albumin ratio (CAR) have been studied as prognostic factors in patients with various cancer types $[9,10]$. Researches showed that T-cell population is predominant in the tumor microenvironment, compared with other inflammatory cells such as natural killer. As referred above, tumor-iniltrating $T$ cells in advanced lung cancer could cause malignancy-induced immunosuppression, which probably weaken the antitumor effect of targeted therapy for advanced NSCLC. In addition, several 
reports revealed that high platelet counts in the baseline was closely associated with shorter overall survival (OS) in advanced NSCLC $[11,12]$. Although PLR was extensively investigated in different tumor categories before, there were rare studies concerning the predictive relationship between PLR and efficacy of EGFR targeted therapy.

Therefore, we analyzed the effect of pretreatment PLR in NSCLC patients with EGFR-TKIs treatment, in the hope of seeking a predictive factor on survival for targeted therapy in patients with advanced NSCLC.

\section{Methods}

\subsection{Patients}

This is a retrospective study of advanced NSCLC patients with EGFR targeted therapy at Dongguan People's Hospital, Southern Medical University, from May 2014 to December 2017. The inclusion criteria were age $\geq 18$ years old, life expectancy of 4 weeks or more, adequate bone marrow function, with stage IIIB (with pleural effusion) and stage IV NSCLC (The International Association for the Study of Lung Cancer 7th edition of Tumor Node Metastasis Staging classification), harboring EGFR gene mutation and never receiving antitumor treatment. EGFR mutations were identified in tumor tissues using the standard sequencing methods. Patients were excluded from the study if they met the following criteria: allergic to targeted therapeutic drugs, primary organ failure, during the pregnancy, hematological or autoimmune disease, serious dysfunction of liver or kidney and unavailable follow-up data. Based on the exclusion criteria, a total of 96 Chinese patients were included in this study. The clinical data of patients in our studies were collected carefully. The local ethics committee approved the study protocol, which was conducted according to the Declaration of Helsinki. Patients provided informed written consent.

\subsection{Clinical management}

All patients with advanced NSCLC in the study were treatment with EGFR-TKIs on a standard dose, including gefitinib, erlotinib and icotinib. The following evaluation was conducted about 3 days before initial treatment: detailed clinical history, physical examination, radiographic results, pathological diagnosis and laboratory parameters including complete blood cell counts and albumin levels. Patients received targeted therapy daily, before unendurable toxicity or disease progression occurred. All the patients were followed up at least 6 months from the initiation of EGFR-TKIs therapy. Computed tomography, radionuclide bone scan and magnetic resonance imaging were conducted to evaluated the treatment efficacy. Tumor response was evaluated according to Response Evaluation Criteria in Solid Tumors (RECIST) criteria. Disease control was defined as complete response (CR), partial response (PR), stable disease (SD) or progression disease (PD). Toxicities were recorded in the light of the National Cancer Institute Common Terminology Criteria for Adverse Events (NCl-CTCAE) version 3.0.

\subsection{Statistical Analysis}


All statistical analyses were performed using the Statistical Product and Service Solutions (SPSS) software, version 22.0 (SPSS, Inc, Chicago, IL). We selected the cut-off value for PLR by using receiver operating characteristic (ROC) curve analysis. The associations among PLR and the clinicopathological

parameters were assessed via Pearson's chi-square test. PFS was defined as time between the start of the treatment and disease progression or death, with censoring for patients alive without progression at last contact. The cutoff date for PFS data was June 28, 2018. By that time, enough data was collected to analyze the efficacy and toxicities of targeted therapy. Objective response rate (ORR) and disease control rate (DCR) were also recorded. Estimates of PFS were calculated using the Kaplan-Meier method and twosided 95\% confidence interval were obtained. Two-sided Log-rank test was used to compare PFS between different PLR groups. A prognostic analysis was carried out using univariate and multivariate Cox regressions models. A two-sided $\mathrm{P}<0.05$ was considered statistically significant.

\section{Results}

\subsection{Patient Characteristics}

As shown in Table 1, all the clinical characteristics were comparable between patients with targeted therapy grouped by PLR. A total of 96 patients with cytological or histological confirmed NSCLC were enrolled in this study. The median age at the time of diagnosis was 61 years (range, 27 to 83 years) and $58.3 \%$ patients were women. Most patients had a performance status of $0-2$ score $(90.6 \%)$ and had sensitive EGFR mutations (95.8\%). The follow-up period ranged from 5.1 to 49.2 months (median, 21.7 months). At the end of the last follow-up, 83 patients had tumor progression. The 1-year PFS rate was $33.7 \%$ for the whole study population.

The mean levels of PLR and albumin were 198 (range 53 to 489 ) and $36.9 \mathrm{~g} / \mathrm{L}$ (range $25.1-45 \mathrm{~g} / \mathrm{L}$ ), respectively. According to the ROC curves, the best cut-off points for the PLR were 190, corresponding to maximum joint sensitivity and specificity. For the PLR, the areas under the ROC curve for PFS was 0.667 and the sensitivity(specificity) was $64.7 \%$ (64.4\%) (Figure 1). Based on the cut-off value of 190, a total of 51 patients $(53.1 \%)$ were detected with high pretreatment PLR $(\geq 190)$. Patients were also divided into subgroups for serum albumin ( $<40$ versus $\geq 40 \mathrm{~g} / \mathrm{L}$ ) according to its upper limit level. The relationship between clinicopathological parameter and pretreatment PLR of patients with EGFR mutated NSCLC is shown in Table 1. There were no statistically significant differences between the two PLR groups.

\subsection{Prognostic Factors}

The patient cohort was divided into high-PLR $(\geq 190)$ and low-PLR $(<190)$ groups using the cut-off value. There was no significant difference in ORR or DCR between the two PLR groups ( $P$ [ 0.05, Table 2). However, Kaplan-Meier analysis showed that patients treated with EGFR-TKIs in the PLR $\geq 190$ group had significantly shorter PFS than those in the PLR $<190$ group $(P=0.009$, Table 2$)$. The 1-year PFS rate in the PLR $\geq 190$ group were lower than the counterpart group (42.2\% versus $19.6 \%)(P=0.016$, Table 2$)$. Further analyses were performed to demonstrate whether the PLR is an independent predictor for PFS in NSCLC patients treated with EGFR-TKIs. 
In univariate analysis, the PLR $(P=0.011)$, pleural effusion $(P=0.026)$ and albumin $(P=0.001)$ were significantly associated with PFS (Table 3 ). In the multivariate Cox regression model, the PLR (HR: 1.781; 95\% Cl: $1.123-2.825 ; \mathrm{P}=0.014$ ) and albumin (HR: $0.388 ; 95 \% \mathrm{Cl}: 0.21-0.715 ; \mathrm{P}=0.002$ ) were significantly associated with PFS, whereas pleural effusion did not show predictive effective to PFS (Table 3). The Kaplan-Meier PFS curves for patients with EGFR-TKIs stratified into PLR and albumin groups were shown in Figures 2 ( $A$ and $B)$.

\subsection{PLR and Toxicities.}

Main toxicities possibly related to EGFR targeted therapy are listed in Table 4. Adverse events of the two PLR groups were generally mild. The most common grade $1 / 2$ adverse events of both groups were nonhematologic toxicities, including rash, raised aminopherase, anorexia and fatigue. As to grade $1 / 2$ adverse events, there was no statistical difference between the two groups. However, For grade 3/4 adverse events of diarrhea, there were significant statistical difference between the two PLR groups. There were 7 episodes of grade $3 / 4$ diarrhea in the PLR $\geq 190$ group, while that in the PLR $<190$ group was only 1 episodes $(P=0.042$, Table 4$)$.

\section{Discussion}

Targeted therapy is the recommended treatment for patients with EGFR mutated advanced NSCLC. The efficacy and toxicity is closely related to the status of EGFR mutation. So far, there is no other superior predictive factor for targeted therapy except EGFR applied extensively in the clinical. Previous studies have already investigated several biomarkers to predict the prognosis of NSCLC, such as PLR, neutrophil count and C-reactive protein/albumin ratio. However, the predictive role of these biomarkers is uncertain in the setting of precision medicine. Therefore, we conducted the present study, with the aim to identify a helpful predictive factor for targeted treatment in advanced NSCLC patients.

Chronic inflammation is involved in cancer formation and progression. PLR is a reproducible and inexpensive hematological marker that was suggested to be a marker of thrombotic and inflammatory conditions $[13,14]$. As known to us, elevated pretreatment PLR in peripheral blood was an independent prognostic factors for various cancers, including advanced NSCLC $[15,16]$. One study showed that pretreatment high PLR were associated with poor survival rates with NSCLC. Nonetheless, there was not impact on the response to chemoradiotherapy [17]. Another study revealed that PLR was a prognostic markers in patients with metastatic NSCLC treated with nivolumab independently of other prognostic factors [18]. Till date, it is still unknown whether PLR is a predictive factor for patient treated with EGFRTKIs.

In our study, we found that pretreatment PLR is significantly associated to the PFS of NSCLC patients with EGFR targeted therapy. Patients in the PLR $\geq 190$ group had shorter PFS than those in the PLR $<190$ group $(P=0.009)$. Furthermore, the 1 -year PFS rate in the PLR $\geq 190$ group were inferior to the low value group $(P=0.016)$. Multivariate analysis confirmed the role of PLR on predicting the efficacy of targeted therapy for advanced NSCLC. In addition, we found that PLR was also an predictive biomarker for grade 
3/4 adverse events of diarrhea. Since diarrhea is the majority of toxicity of EGFR targeted therapy, it maybe be important to adopt prophylactic measures for those patients with high pretreatment PLR. In this study, we found that hypoalbuminemia is negatively associated with the efficacy of EGFR therapy, which is in line with the results of former reports concerning several types of cancer. While the number of patients in this study was relatively small, further studies are needed to illuminate the relationship between PLR and EGFR-TKIs treatment.

Our study show that PLR is a superior independent prognostic factor in advanced patients with EGFR mutated lung cancer. In the recent decade, only EGFR mutation is an effective predictive biomarker for efficacy and toxicity in targeted therapy such as gefitinib and erlotinib $[19,20]$. Recent studies never considered the crucial impact of pretreatment PLR on therapeutic outcomes in patients receiving EGFRTKIs, as compared in that of chemotherapy and immunotherapy of advanced NSCLC. This study revealed the reality that pretreatment PLR and albumin level could be an beneficial factor to predict the result of targeted therapy for NSCLC patients.

Nonetheless, this study still had several limitations. Firstly, this is a retrospective analysis with a relatively small number of NSCLC patients. Hence, comprehensive multivariable analyses was not allowed in the study. Secondly, it was inevitably affected by residual confounding factors such as NLR, CAR and hyperfibrinogenemia. Thirdly, there may have been elevated risk of a patient selection bias in the study, as our study is a single center investigation. Finally, the EGFR targeted therapy contained four types of antitumor agents, although previous studies proved that these drugs had almost the same efficacy $[21,22]$. However, the correlation of high pretreatment PLR and poor efficacy was still statistical significance and of vital importance in the clinical.

In conclusion, our study showed that high pretreatment PLR was an independent indicator for predicting a poor PFS for NSCLC patients receiving EGFR-TKIs treatment. Further studies are needed to identify the impact of pretreatment PLR on results of EGFR-TKIs treatment. Translational research is suggested to further investigate the mechanism of this clinical finding.

\section{Declarations}

\section{Compliance with ethical standards}

Conflict of interest: The authors declare that they have no conflict of interest.

Ethical approval: All procedures performed in studies involving human participants were in accordance with the ethical standards of the institutional and/or national research committee and with the 1964 Helsinki 
declaration and its later amendments or comparable ethical standards.

This article does not contain any animal studies performed by any of

the authors.

Informed consent: Informed consent was obtained from all individual

participants included in the study.

\section{References}

1. Siegel RL, Miller KD, Jemal A. Cancer Statistics, 2017. CA Cancer J Clin. 2017;67:7-30.

2. Liu KJ, Wu HY. A retrospective analysis of cisplatin, pemetrexed, and bevacizumab in previously treated non-small-cell lung cancer. Oncotarget. 2015 Sep 8;6(26):22750-7.

3. Chen F, Cole P, Bina WF. Time trend and geographic patterns of lung adenocarcinoma in the united states, 1973-2002. Cancer Epidemiol Biomarkers Prev 2007;16:2724-2729.

4. Rosell R, Carcereny E, Gervais R, et al, on behalf of the Spanish Lung Cancer Group in collaboration with the Groupe Français de Pneumo-Cancérologie and the Associazione Italiana Oncologia Toracica. Erlotinib versus standard chemotherapy as first-line treatment for European patients with advanced EGFR mutation-positive non-small-cell lung cancer (EURTAC): a multicentre, open-label, randomised phase 3 trial. Lancet Oncol 2012; 13: 239-46.

5. Shigematsu H, Lin L, Takahashi T, et al. Clinical and biological features associated with epidermal growth factor receptor gene mutations in lung cancers. J Natl Cancer Inst 2005; 97: 339-46.

6. Gomes M, Teixeira AL, Coelho A, Araújo A, Medeiros R. The role of inflammation in lung cancer. Adv Exp Med Biol. 2014;816:1-23.

7. Shiels MS, Pfeiffer RM, Hildesheim A, Engels EA, Kemp TJ, Park JH, Katki HA, Koshiol J, Shelton G, Caporaso NE, Pinto LA, Chaturvedi AK. Circulating inflammation markers and prospective risk for lung cancer. J Natl Cancer Inst. 2013;105:1871-80.

8. Mantovani A, Allavena P, Sica A, Balkwill F. Cancer-related inflammation. Nature. 2008; 454:436-44.

9. Zhang WW, Liu KJ, Hu GL, Liang WJ. Preoperative platelet/lymphocyte ratio is a superior prognostic factor compared to othersystemic inflammatory response markers in ovarian cancer patients. Tumour Biol. 2015;36:8831-7.

10. Zhang WW, Liu KJ, Ye B, Liang WJ, Ren YZ. Pretreatment C-reactive protein/albumin ratio is associated with poor survival in patients with stage IB-IIA cervical cancer. Cancer Med. 2018;7:105-113. 
11. Gonzalez Barcala FJ, Garcia Prim JM. Platelet count: association with prognosis in lung cancer. Med Oncol. 2010; 27: 357-62.

12. Aoe K, Hiraki A, Ueoka H. Thrombocytosis as a useful prognostic indicator in patients with lung cancer. Respiration. 2004; 71: 170-3.

13. Smith RA, Ghaneh P, Sutton R, Raraty M, Campbell F, Neoptolemos JP. Prognosis of resected ampullary adenocarcinoma by preoperative serum CA19-9 levels and platelet-lymphocyte ratio. $J$ Gastrointest Surg. 2008;12:1422-8.

14. Wang D, Yang JX, Cao DY, Wan XR, Feng FZ, Huang HF, Shen K, Xiang Y. Preoperative neutrophillymphocyte and platelet-lymphocyte ratios as independent predictors of cervical stromal involvement in surgically treated endometrioid adenocarcinoma. Onco Targets Ther. 2013;6:211-6.

15. Guthrie GJ, Charles KA, Roxburgh CS, Horgan PG, McMillan DC, Clarke SJ. The systemic inflammation-based neutrophil-lymphocyte ratio: experience in patients with cancer. Crit Rev Oncol Hematol. 2013;88:218-30.

16. Templeton AJ, Ace O, McNamara MG, Al-Mubarak M, Vera-Badillo FE, Hermanns T, Seruga B, Ocaña A, Tannock IF, Amir E. Prognostic role of platelet to lymphocyte ratio in solid tumors: a systematic review and meta-analysis. Cancer Epidemiol Biomarkers Prev. 2014;23:1204-12.

17. Unal D, Eroglu C, Kurtul N, Oguz A, Tasdemir A. Are neutrophil/lymphocyte and platelet/lymphocyte rates in patients with non-small cell lungcancer associated with treatment response and prognosis? Asian Pac J Cancer Prev. 2013;14:5237-42.

18. Diem S, Schmid S, Krapf M, Flatz L, Born D, Jochum W, Templeton AJ, Früh M. Neutrophil-toLymphocyte ratio (NLR) and Platelet-to-Lymphocyte ratio (PLR) as prognosticmarkers in patients with non-small cell lung cancer (NSCLC) treated with nivolumab. Lung Cancer. 2017;111:176-181.

19. Perez-Soler R, Chachoua A, Hammond LA, Rowinsky EK, Huberman M, Karp D, Rigas J, Clark GM, Santabárbara P, Bonomi P. Determinants of tumor response and survival with erlotinib in patients with non--small-cell lung cancer. J Clin Oncol. 2004; 22:3238-3247.

20. Kris MG, Natale RB, Herbst RS, Lynch TJ Jr, Prager D, Belani CP, Schiller JH, Kelly K, Spiridonidis H, Sandler A, Albain KS, Cella D, Wolf MK, Averbuch SD, Ochs JJ, Kay AC. Efficacy of gefitinib, an inhibitor of the epidermal growth factor receptor tyrosine kinase, in symptomatic patients with non-small cell lung cancer: a randomized trial. JAMA. 2003; 290:2149-2158.

21. Burotto M, Manasanch EE, Wilkerson J, Fojo T. Gefitinib and erlotinib in metastatic non-small cell lung cancer: a meta-analysis of toxicity and efficacy of randomized clinical trials. Oncologist. 2015;20(4):40010. 
22. Shi YK, Zhang L, Liu XQ, Zhou CC, Zhang L, Zhang SC, Wang D, Li Q, Qin SK, Hu CH, Zhang YP, Chen JH, Cheng Y, Feng JF, Zhang HL, Song Y, Wu YL, Xu N, Zhou JY, Luo RC, Bai CX, Jin YN, Liu WC, Wei ZH, Tan FL, Wang YX, Ding LM, Dai H, Jiao SC, Wang J, Liang L, Zhang WM, Sun Y. Icotinib versus gefitinib in previously treated advanced non-small-cell lung cancer (ICOGEN): a randomised, double-blind phase 3 non-inferiority trial. Lancet Oncol. 2013;14(10):953-61.

\section{Tables}

Table 1. Correlations between PLR and patient characteristics before targeted therapy.

\begin{tabular}{|c|c|c|c|c|}
\hline Characteristic & Case $(n=96)$ & PLR $<190(n=45)$ & PLR $\geq 190(n=51)$ & $P$ value \\
\hline \multicolumn{5}{|l|}{ Age } \\
\hline$<65$ & $57(59.4 \%)$ & $28(62.2 \%)$ & $29(56.9 \%)$ & 0.59 \\
\hline$\geq 65$ & $39(40.6 \%)$ & $17(37.8 \%)$ & $22(43.1 \%)$ & \\
\hline \multicolumn{5}{|l|}{ Sex } \\
\hline Male & $40(41.7 \%)$ & $17(37.8 \%)$ & $2345.1 \%)$ & 0.47 \\
\hline Female & $56(58.3 \%)$ & $28(62.2 \%)$ & $28(54.9 \%)$ & \\
\hline \multicolumn{5}{|l|}{ ECOG PS } \\
\hline$<2$ & $71(73.9 \%)$ & $32(71.1 \%)$ & $39(76.5 \%)$ & 0.55 \\
\hline$\geq 2$ & $25(26.1 \%)$ & $13(28.9 \%)$ & $12(23.5 \%)$ & \\
\hline \multicolumn{5}{|l|}{ Tumor location } \\
\hline Left & $41(42.7 \%)$ & $23(51.1 \%)$ & $18(35.3 \%)$ & 0.12 \\
\hline Right & $55(57.3 \%)$ & $22(48.9 \%)$ & $33(64.7 \%)$ & \\
\hline \multicolumn{5}{|l|}{ Smoking } \\
\hline Yes & $21(21.9 \%)$ & $9(20 \%)$ & $11(21.6 \%)$ & 0.85 \\
\hline No & $75(78.1 \%)$ & $36(80 \%)$ & $40(78.4 \%)$ & \\
\hline \multicolumn{5}{|l|}{ Metastasis } \\
\hline$<3$ & $53(55.2 \%)$ & $27(60 \%)$ & $26(50.9 \%)$ & 0.38 \\
\hline$\geq 3$ & $43(44.8 \%)$ & $18(40 \%)$ & $25(49.1 \%)$ & \\
\hline \multicolumn{5}{|l|}{ Brain metastasis } \\
\hline Yes & $38(39.6 \%)$ & $21(46.7 \%)$ & $17(33.3 \%)$ & 0.18 \\
\hline No & $58(60.4 \%)$ & $24(53.3 \%)$ & $34(66.7 \%)$ & \\
\hline \multicolumn{5}{|l|}{ Pleural effusion } \\
\hline No & $44(45.8 \%)$ & $24(53.3 \%)$ & $20(39.2 \%)$ & 0.17 \\
\hline Yes & $52(54.2 \%)$ & $21(46.7 \%)$ & $31(60.8 \%)$ & \\
\hline \multicolumn{5}{|l|}{ BMI } \\
\hline$<24$ & $64(66.7 \%)$ & $29(64.4 \%)$ & $35(68.6 \%)$ & 0.66 \\
\hline$\geq 24$ & $32(33.3 \%)$ & $16(35.6 \%)$ & $16(31.4 \%)$ & \\
\hline \multicolumn{5}{|l|}{ Albumin (g/L) } \\
\hline$<40$ & $75(78.1 \%)$ & $33(73.3 \%)$ & $42(82.4 \%)$ & 0.29 \\
\hline$\geq 40$ & $21(21.9 \%)$ & $12(26.7 \%)$ & $9(17.6 \%)$ & \\
\hline
\end{tabular}


Table 2. Efficacy Results of patients with different PLR.

\begin{tabular}{|c|c|c|l|}
\hline & PLR $<190(\mathrm{n}=45)$ & PLR $\geq 190(\mathrm{n}=51)$ & \\
\hline Variable & No. $\%$ & No. $\%$ & \\
\hline Response & \multicolumn{1}{|c|}{ P value } \\
\hline PR (\%) & 2862.2 & $27 \quad 52.9$ & \\
\hline SD (\%) & $13 \quad 28.9$ & $14 \quad 27.5$ & \\
\hline PD (\%) & $4 \quad 8.9$ & $10 \quad 19.6$ & \\
\hline Response rates, \% & 62.2 & 52.9 & 0.36 \\
\hline 95\% Cl & 37.3 to 67.2 & 44.1 to 69.8 & \\
\hline Disease control rates, \% & 91.1 & 80.4 & 0.14 \\
\hline 95\% Cl & 70.3 to 93.3 & 79.5 to 96.4 & \\
\hline Median PFS (months) & 12.4 & 6.6 & 0.009 \\
\hline 95\% Cl & 9.5 to 15.4 & 4.8 to 8.4 & \\
\hline 1 year PFS rates (\%) & 42.2 & 19.6 & 0.016 \\
\hline
\end{tabular}

Table 3. Univariate and multivariate analyses of PFS in advanced NSCLC patients. 


\begin{tabular}{|c|c|c|c|c|}
\hline Variable & $\begin{array}{c}\text { Univariate } \\
\text { HR }(95 \% \mathrm{Cl})\end{array}$ & $P$ value & Multivariate $\mathrm{HR}(95 \% \mathrm{Cl})$ & $P$ value \\
\hline \multicolumn{5}{|l|}{ Age } \\
\hline$<65$ & 1 & & & \\
\hline$\geq 65$ & $0.717(0.458-1.122)$ & 0.145 & & \\
\hline \multicolumn{5}{|l|}{ Sex } \\
\hline Male & 1 & & & \\
\hline Female & $1.153(0.742-1.792)$ & 0.526 & & \\
\hline \multicolumn{5}{|l|}{ ECOG PS } \\
\hline$<2$ & 1 & & & \\
\hline$\geq 2$ & $1.134(0.68-1.893)$ & 0.629 & & \\
\hline \multicolumn{5}{|c|}{ Tumor location } \\
\hline Left & 1 & & & \\
\hline Right & $1.5(0.956-2.352)$ & 0.078 & & \\
\hline \multicolumn{5}{|l|}{ Smoking } \\
\hline Yes & 1 & & & \\
\hline No & $1.18(0.68-2.047)$ & 0.555 & & \\
\hline \multicolumn{5}{|l|}{ Metastasis } \\
\hline$<3$ & 1 & & & \\
\hline$\geq 3$ & $1.4(0.898-2.183)$ & 0.137 & & \\
\hline \multicolumn{5}{|c|}{ Brain metastasis } \\
\hline Yes & 1 & & & \\
\hline No & $0.741(0.474-1.159)$ & 0.189 & & \\
\hline \multicolumn{5}{|c|}{ Pleural effusion } \\
\hline No & 1 & & 1 & \\
\hline Yes & $1.649(1.061-2.562)$ & 0.026 & $1.185(0.748-1.879)$ & 0.469 \\
\hline \multicolumn{5}{|l|}{$\overline{\mathrm{BMI}}$} \\
\hline$<24$ & 1 & & & \\
\hline$\geq 24$ & $1.401(0.889-2.207)$ & 0.146 & & \\
\hline \multicolumn{5}{|c|}{ Albumin (g/L) } \\
\hline$<40$ & 1 & & 1 & \\
\hline$\geq 40$ & $0.376(0.208-0.678)$ & 0.001 & $0.388(0.21-0.715)$ & 0.002 \\
\hline \multicolumn{5}{|l|}{ PLR } \\
\hline$<190$ & 1 & & 1 & \\
\hline$\geq 190$ & $1.795(1.147-2.811)$ & 0.011 & $1.781(1.123-2.825)$ & 0.014 \\
\hline
\end{tabular}

Table 4. Treatment related toxicities of patients with different PLR. 


\begin{tabular}{|c|c|c|c|c|c|c|}
\hline & \multicolumn{2}{|c|}{ Grade 1/2 } & \multirow{3}{*}{$P$ value } & \multicolumn{2}{|c|}{ Grade 3/4 } & \multirow[b]{3}{*}{$P$ value } \\
\hline Toxicity & PLR $<190(n=45)$ & PLR $\geq 190(n=51)$ & & PLR < $190(n=45)$ & PLR $\geq 190(n=51)$ & \\
\hline & & & & & & \\
\hline Rash & 12 & 17 & 0.483 & 4 & 7 & 0.463 \\
\hline Pruritus & 6 & 8 & 0.748 & 0 & 0 & \\
\hline Dizziness & 3 & 8 & 0.17 & 0 & 0 & \\
\hline Fever & 3 & 7 & 0.263 & 0 & 0 & \\
\hline Diarrhea & 9 & 5 & 0.161 & 1 & 7 & 0.042 \\
\hline Fatigue & 6 & 12 & 0.206 & 0 & 2 & 0.183 \\
\hline Nausea & 7 & 10 & 0.608 & 0 & 0 & \\
\hline Vomiting & 9 & 7 & 0.416 & 0 & 0 & \\
\hline Anorexia & 10 & 19 & 0.112 & 0 & 0 & \\
\hline Raised aminopherase & 12 & 18 & 0.368 & 1 & 0 & \\
\hline & & & & & & 0.289 \\
\hline Dyspnea & 6 & 6 & 0.819 & 1 & 4 & 0.22 \\
\hline Hemorrhage & 1 & 5 & 0.128 & 0 & 1 & 0.35 \\
\hline
\end{tabular}

Figures 


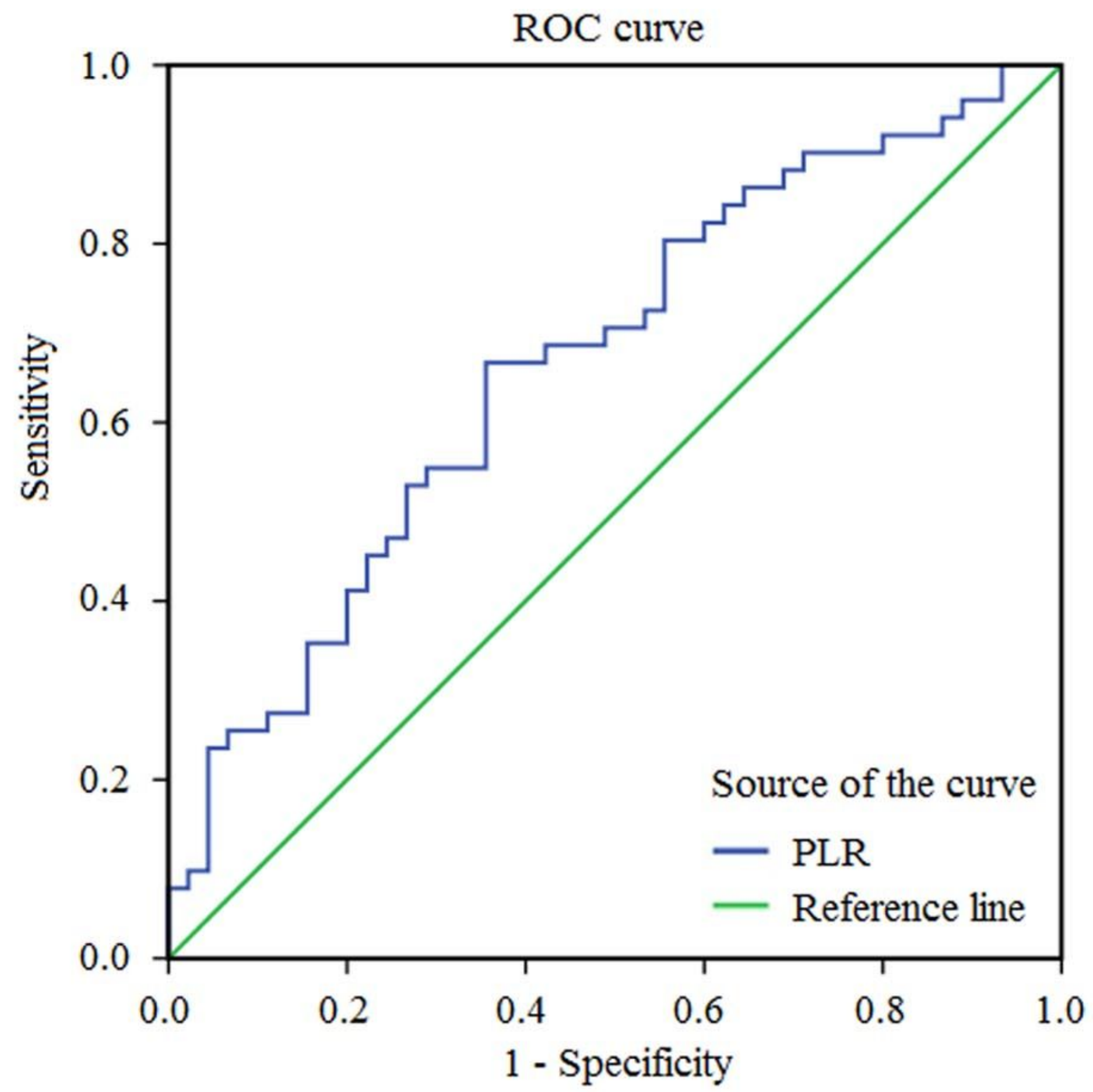

Figure 1

Receiver operating characteristic curves for pretreatment PLR for PFS. 
A

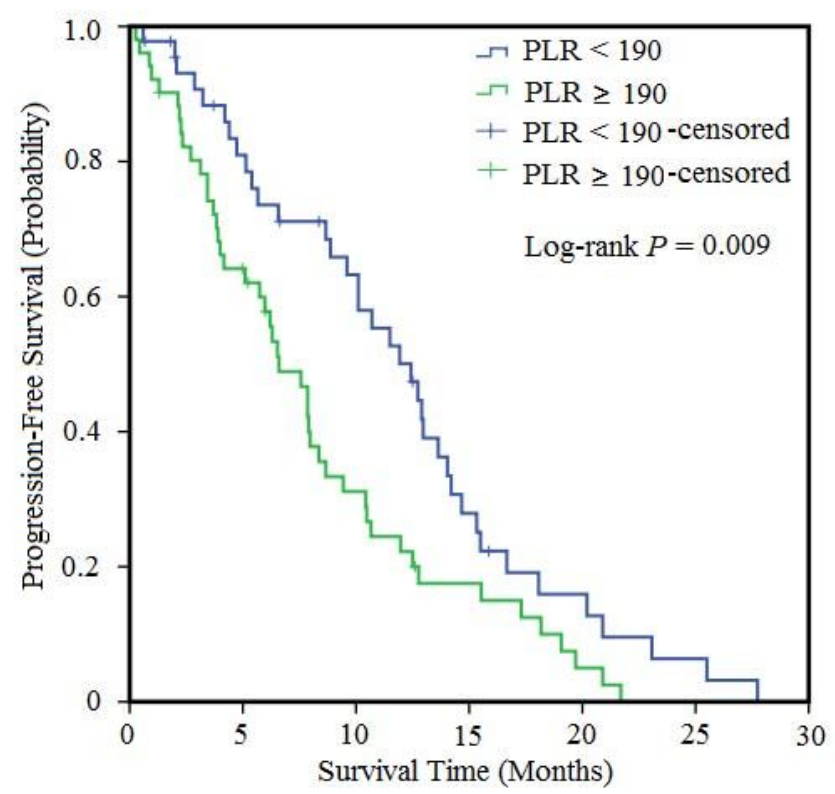

B

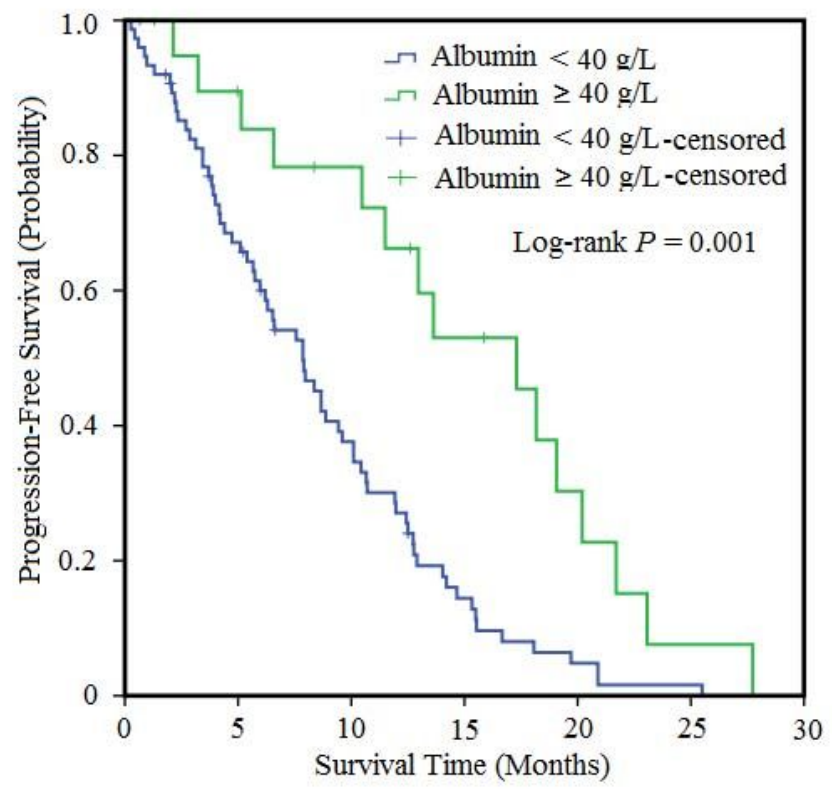

Figure 2

Kaplan-Meier curves for PFS of different pretreatment PLR (A) and albumin level (B). 